\title{
Cinética de secagem da polpa cupuaçu (Theobroma grandiflorum) pré desidratada por imersão-impregnação ${ }^{1}$
}

\author{
Kinetic drying of cupuaçu pulp (Theobroma grandiflorum) pre-dehydrated by \\ immersion-impregnation
}

\author{
Luty Gomezcaceres Perez ${ }^{2}$, Francisca Marta Nascimento de Oliveira ${ }^{3 *}$, Jerusa Souza Andrade ${ }^{4}$ e Mario Moreira \\ Filho $^{5}$
}

\begin{abstract}
RESUMO - A desidratação osmótica é uma operação unitária importante para transformar os frutos perecíveis em novos produtos com valor agregado e com maior vida de prateleira. A aplicação dos modelos matemáticos confiáveis permite prever o comportamento dos diversos fenômenos que ocorrem durante o processo de secagem, o que implica na redução do custo operacional. A polpa do cupuaçu é comumente usada para preparar sucos e sorvetes, mas durante a safra é produzida em grande quantidade, que muitas vezes, é descartada devido ao custo de produção ser mais alto do que o da comercialização. O objetivo desse estudo foi investigar o efeito da solução binária e da ternária na taxa da secagem por convecção e avaliar a adequação dos modelos matemáticos na descrição das curvas da secagem do cupuaçu. No experimento, a pré-desidratação foi feita com solução binária (sacarose a $40{ }^{\circ}$ Brix) e ternária $(80 \%$ de sacarose $/ 20 \%$ de cloreto de sódio), durante três horas em temperatura ambiente, seguida pela secagem convencional na estufa (ar circulante) a $65^{\circ} \mathrm{C}$, até peso constante. Os dados experimentais foram ajustados utilizando os modelos de Page e Midilli, Kucuk e Yapar. A solução binária usada na pré-secagem reduziu o tempo da subseqüente secagem convencional. Com variação de 99,64 a 99,77\% no coeficiente de determinação $\left(R^{2}\right)$, o modelo de Page apresentou o melhor ajuste aos dados experimentais.
\end{abstract}

Palavras-chave: Cupuaçu. Alimentos desidratados. Modelos matemáticos.

\begin{abstract}
The osmotic dehydration is an important unitary operation to transform the perishable fruits in new products with added value and longer shelf life. The application of reliable mathematical models allows prediction the behavior of various phenomena that occur during the drying process, which implies in reduction of operating cost. The cupuaçu pulp is commonly used to prepare juices and ice creams, but during the crop it is produced in large quantity, which is often discarded because of the cost of production is higher than the selling price. The aim of this study was to investigate the effect of binary and ternary solutions at the rate of convective drying and evaluate the adequacy of mathematical models to describe the drying curves of the cupuaçu. In the experiment, osmotic dehydration was carried out with binary (sucrose at $40^{\circ} \mathrm{Brix}$ ) and ternary ( $80 \%$ sucrose $/ 20 \%$ sodium chloride) solutions for three hours at room temperature, followed by conventional drying in an oven (air-circulating) at $65{ }^{\circ} \mathrm{C}$ to constant weight. The experimental data were adjusted using the models of Page and Midilli, Kucuk and Yapar. The binary solution used during predrying, reduced the time of the subsequent conventional drying. Varying from 99.64 to $99.77 \%$ in the coefficient of determination $\left(\mathrm{R}^{2}\right)$, the Page model showed a better adjustment to experimental data.
\end{abstract}

Key words: Cupuassu. Dehydrated food. Mathematical models.

\footnotetext{
*Autor para correspondência

${ }^{1}$ Recebido para publicação em 06/05/2010; aprovado em 24/05/2012

Pesquisa financiada pela FINEP

${ }^{2}$ Departamento de Engenharia Industrial, Faculdade de Engenharia, Corporación Universitaria del Caribe, Sincelejo, Colômbia, lutygo@ hotmail.com ${ }^{3}$ Coordenação de Graduação, Universidade Nilton Lins, Avenida Professor Nilton Lins, No 3.259, Parque das Laranjeiras, Manaus-AM, Brasil, 69.058-030,fmartaagri@ hotmail.com

${ }^{4}$ Departamento de Tecnologia de Alimentos, Instituto Nacional de Pesquisas da Amazônia/Coordenação de graduação, Universidade Nilton Lins, Manaus-AM, Brasil, andrade@inpa.gov.br

${ }_{5}^{5}$ Programa PCI-INPA, Departamento de Ciências Agronômicas, Instituto Nacional de Pesquisas da Amazônia, Manaus-AM, Brasil, rutaceae@ yahoo.com.br
} 


\section{INTRODUÇÃO}

O cupuaçueiro (Theobroma grandiflorum Schum) é nativo da Região amazônica e sua polpa apresenta características ideais para o aproveitamento industrial (COSTA et al., 2003) sendo utilizada no preparo de sorvetes, sucos, geléias e diversas sobremesas (QUEIROZ; FIGUEIRÊDO; CABRAL, 2004). Porém, para o produtor rural o valor de comercialização é baixo, equiparando com o custo de produção, pois o uso do congelamento para a conservação da polpa ainda é o método utilizado na região. Portanto, é fundamental a busca de novos produtos que possam agregar valor a polpa do fruto.

A desidratação é utilizada na preservação dos alimentos, por reduzir a quantidade de água livre, responsável pelas principais causas de deterioração nos alimentos (ANDRADE et al., 2003). A desidratação Osmótica (D. O.) consiste na imersão dos produtos em soluções hipertônicas de um ou mais solutos, originando dois fluxos simultâneos e opostos (TONOM; BARONI; HUBINGER, 2006). A escolha do tipo e da concentração do agente desidratante depende do produto desejado, das alterações ocasionadas nas características do produto final e do custo do processo (LENART, 1996).

A desidratação osmótica reduz a atividade de água, inibe a atividade enzimática e retêm a cor natural, além de originar produtos novos (FITO et al., 2001). No entanto, apenas a D. O. não consegue diminuir a umidade do material a um nível que garanta estabilidade durante estocagem em temperatura ambiente, sendo necessário a secagem complementar (SERENO et al., 2001). A combinação desses dois processos permite a obtenção de desidratados que conservam características sensoriais e nutricionais mais próximas do fruto in natura (CORRÊA et al., 2008; MASTRANTONIO; PEREIRA; HUBINGER, 2006).

A secagem por convenção é um processo complexo que envolve transferência de calor e massa. Durante o processo o calor evapora a água do alimento e o ar remove o vapor d'água, reduzindo a umidade e a atividade de água e aumentando a vida útil do produto final (FIOREZE, 2004). Para melhorar e controlar esse processo são utilizados modelos que simulam as curvas de secagem nas diversas condições (KARATHANOS; BELESSIOTIS, 1999). Atualmente, existem diversos estudos sobre o fenômeno do transporte de massa em alimentos, mas ainda há muitas dúvidas decorrentes da complexidade desses sistemas, sendo imprescindível conhecer as características da secagem e os modelos que melhor descrevem o comportamento de cada alimento pesquisado (SARAVACOS; MAROULS, 2001).

Os modelos teóricos que descrevem a taxa decrescente de secagem de um sólido consideram, geralmente, como mecanismo principal, a difusão baseada na segunda Lei de Fick, onde o fluxo de massa por unidade de área é proporcional ao gradiente de concentração de água (PARK; VOHNIKOZA; BROD, 2002, ROMEROPEÑA; KIECKBUSCH, 2003).

Dentre os modelos sugeridos, encontram-se o modelo de Page que é uma simplificação do modelo de Fick, e o modelo semi-empírico (MIDILLI; KUCUK; YAPAR, 2002) obtido experimentalmente a partir do estudo da secagem de cogumelos, pólen e pistache, sendo também, uma simplificação do modelo de Fick (LIMA, FIGUEIREDO; QUEIROZ, 2007). Estes modelos além de permitir uma avaliação dos mecanismos e das propriedades físicas de transporte de massa durante o processo são importantes na seleção e desenvolvimento de equipamentos, e no cálculo dos custos operacionais (DIONELLO et al., 2009).

Diante do exposto, o objetivo deste estudo foi investigar o efeito da pré-desidratação osmótica da polpa de cupuaçu em diferentes soluções na secagem por convecção e avaliar a cinética de secagem por diferentes modelos.

\section{MATERIAL E MÉTODOS}

O trabalho foi desenvolvido no Departamento de Tecnologia de Alimentos do Instituto Nacional de Pesquisas da Amazônia (INPA) utilizando-se polpa de cupuaçu, proveniente de propriedade rural, situada no $\mathrm{Km} 5$ do ramal ZF7, na altura do $\mathrm{Km} 90$ da rodovia AM-10 no município de Rio Preto da Eva (AM).

As polpas foram pesadas e transferidas para cestos (saches) de tela de nylon. Para o experimento foram preparados dois lotes e cada um contendo três cestos correspondendo a triplicata. O primeiro lote foi submetido à desidratação osmótica com solução binária de sacarose a $40{ }^{\circ}$ Brix e o segundo com solução ternária composta de $80 \%$ de sacarose e $20 \%$ de cloreto de sódio durante três horas e em temperatura ambiente $\left(28{ }^{\circ} \mathrm{C}\right)$. Após a desidratação os cestos contendo as polpas foram retirados da imersão, rapidamente lavados com água corrente para remoção da solução aderida na superfície e mantidos sobre bandejas metálicas perfuradas por tempo suficiente para a drenagem da água. Em seguida as polpas foram transferidas para placas de Petri previamente numeradas e pesadas.

Antes e após a secagem foram retiradas amostras para determinação (estufa a $105^{\circ} \mathrm{C}$ até peso constante) dos teores de umidade inicial e final (INSTITUTO ADOLFO LUTZ, 2008). A secagem das polpas foi realizada em estufa com circulação forçada de ar e temperatura de $65^{\circ} \mathrm{C}$ até atingir os pesos constantes. Para o monitoramento da secagem, periodicamente as placas contendo as polpas foram retiradas, pesadas e retornadas para a estufa. Após 
atingir o equilíbrio as amostras foram retiradas e mantidas em temperatura ambiente até o resfriamento. Para o ajuste dos dados experimentais foram utilizados os modelos de Page (Eq. 1) e Midilli, Kucuk e Yapar (Eq. 2), através do programa Statistica 7.0 e de análises de regressão não linear.

$R U=\exp \left(-k t^{n}\right)$

$R U=a \exp \left(-k t^{n}\right)+b t$

em que: RU = razão de umidade (adimensional); k, n, a, b $=$ constantes do modelo; $\mathrm{t}=$ tempo (minutos).

Os critérios para a escolha do melhor ajuste dos modelos aos dados experimentais, foram: o coeficiente de determinação $\left(\mathrm{R}^{2}\right)$ e o erro percentual médio $(\mathrm{P})$ que foi calculado (Eq. 3) para avaliar se os modelos utilizados foram ou não preditivos $(\mathrm{P}<10 \%)$.

$$
P=\frac{100}{N} \sum_{i=1}^{N} \frac{\left|R U_{\text {observado }}-R U_{\text {predito }}\right|}{R U_{\text {observado }}}
$$

em que: $\mathrm{P}=$ Erro percentual médio $(\%) ; \mathrm{RU}_{\text {predito }}=$ razão de umidade predita pelo modelo (adimensional); $\mathrm{RU}_{\text {observado }}=$ razão de umidade experimental (adimensional); $\mathrm{N}=$ número de dados experimentais.

\section{RESULTADOS E DISCUSSÃO}

Com teor inicial de $85,03 \%$ de umidade, após a desidratação osmótica em soluções binária e ternária as polpas de cupuaçu, apresentaram, respectivamente, teores de $70,28 \%$ e $59,95 \%$ de umidade. A redução de $14,75 \%$ (solução binária) e 25,08\% (solução ternária) em relação ao teor inicial tem efeito positivo na redução do tempo da secagem subseqüente. A maior eficiência da solução ternária decorre da combinação dos gradientes criados tanto pelo sal como pela sacarose, que favorece a remoção de uma maior quantidade de água das células. Além disso, a presença de sal inibe a formação da barreira de açúcar, ocasionando também maiores taxas de desidratação (TSAMO et al., 2005; TONOM; BARONI; HUBINGER, 2006).

BARONI(2004), desidratando osmoticamente tomate em soluções binárias e ternárias de sal e açúcar, verificou que a combinação destes solutos favoreceu a remoção de água e diminuiu a penetração de sólidos no produto.

O percentual de perda de peso durante 450 minutos do processo (Figura 1) apresentou valores de coeficiente de determinação de $84,41 \%$ para a solução binária e $42,71 \%$ para a ternária, mostrando que, nessas condições de trabalho, a equação polinomial encontrada para solução binária teve um bom ajuste $\left(\mathrm{R}^{2}>80 \%\right)$.

Os ajustes dos dados experimentais pelo modelo de Page para as polpas de cupuaçu pré desidratadas e
Figura 1 - Percentual de perda de peso durante a secagem em estufa de polpas de cupuaçu previamente desidratadas em solução binária e ternária

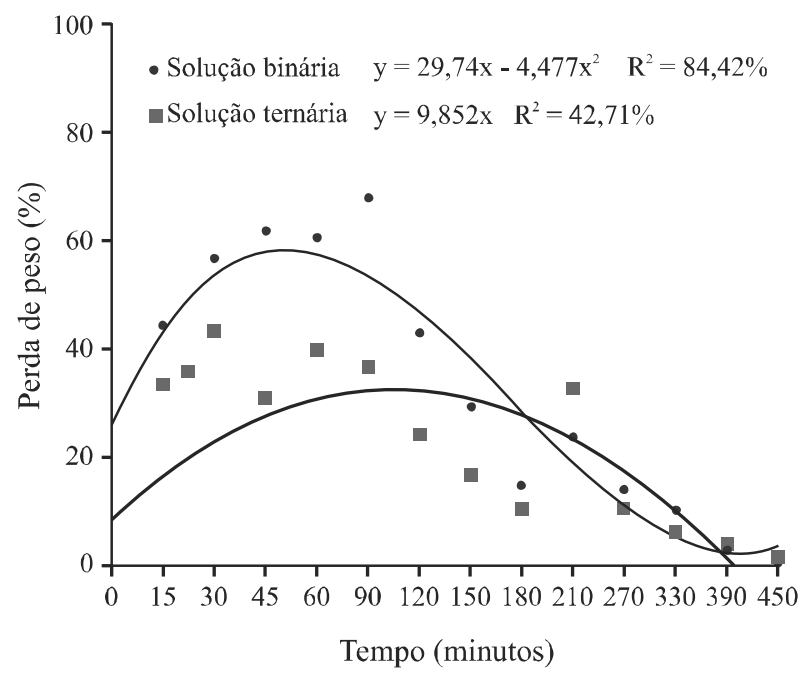

secas em estufa são apresentados na Figura 2. Para ambas as soluções o comportamento das curvas foi semelhante e apresentou um excelente ajuste. No entanto verificase que houve diferença no tempo final da secagem. $\mathrm{O}$ processo de secagem das polpas pré tratadas em solução binária tornou-se constante aos 330 minutos. Na polpa previamente desidratada em solução ternária a razão de umidade tornou-se constante em torno de 400 minutos. Assim, com menor tempo de secagem, o uso de solução binária indica redução no custo com energia elétrica.

Nas curvas, o período de taxa constante não foi observado e o processo de secagem para polpa de cupuaçu acontece na faixa de período de taxa decrescente. Nesse caso a transferência interna de água é quem governa e fixa a taxa de secagem. Resultado semelhante foi observado por Doymaz (2006) na secagem de uvas pretas pré tratadas com diferentes soluções. Para maioria dos frutos o período de taxa decrescente é quase sempre o único observado (EL-AOUAR; AZOUBEL; MURR, 2003; PARK; VOHNIZOK; BROD, 2002).

Na Figura 3 são apresentadas as curvas dos ajustes dos dados experimentais pelo modelo de Midilli, Kucuk e Yapar (2002) para as polpas de cupuaçu pré desidratadas por osmose e secas em estufa. Observa-se que os dados não podem ser estimados por este modelo, pois o ajuste não foi bom.

Alguns autores reportam que existe certa dificuldade em se comparar resultados reportados na literatura, em função dos diferentes métodos de determinação e dos modelos utilizados, e também, pelas diferenças na composição e na estrutura física dos alimentos (ANDRADE et al., 2007, AZOUBEL; MURR, 2004). 
Figura 2 - Cinética de secagem em estufa de polpas de cupuaçu previamente desidratadas em soluções binária e ternária utilizando o modelo de Page para o ajuste dos dados experimentais

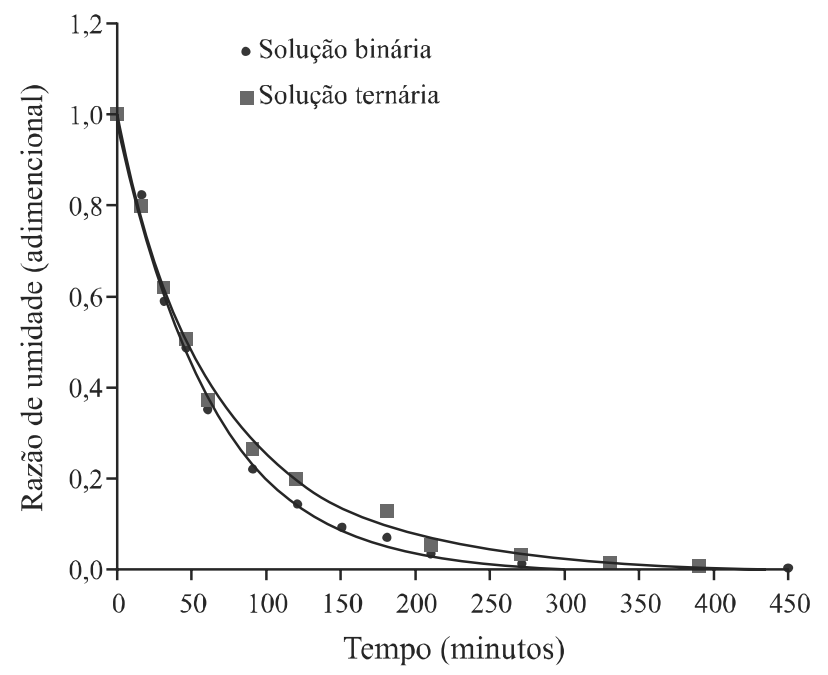

Na Tabela 1 encontram-se os valores dos parâmetros dos modelos de Page e Midilli, Kucuk e Yapar, ajustados aos dados experimentais das cinéticas de secagem das polpas de cupuaçu, os coeficientes de determinação $\left(\mathrm{R}^{2}\right)$ e os erros percentuais médios $(\mathrm{P})$.

Nota-se que o modelo de Page se ajustou bem aos dados experimentais de secagem para os tratamentos estudados, apresentando coeficientes de determinação $\left(\mathrm{R}^{2}\right)$ superiores a $99 \%$ e erros percentuais médios $(\mathrm{P})$ inferiores a $8,5 \%$, podendo ser usados na predição da cinética de secagem da polpa de cupuaçu. Já o modelo de Midilli, Kucuk e Yapar apresentou valores P superiores a 70\% não podendo representar o processo.
Figura 3 - Cinética de secagem em estufa de polpas de cupuaçu previamente desidratadas em soluções binária e ternária utilizando o modelo de Midilli, Kucuk e Yapar para o ajuste dos dados experimentais

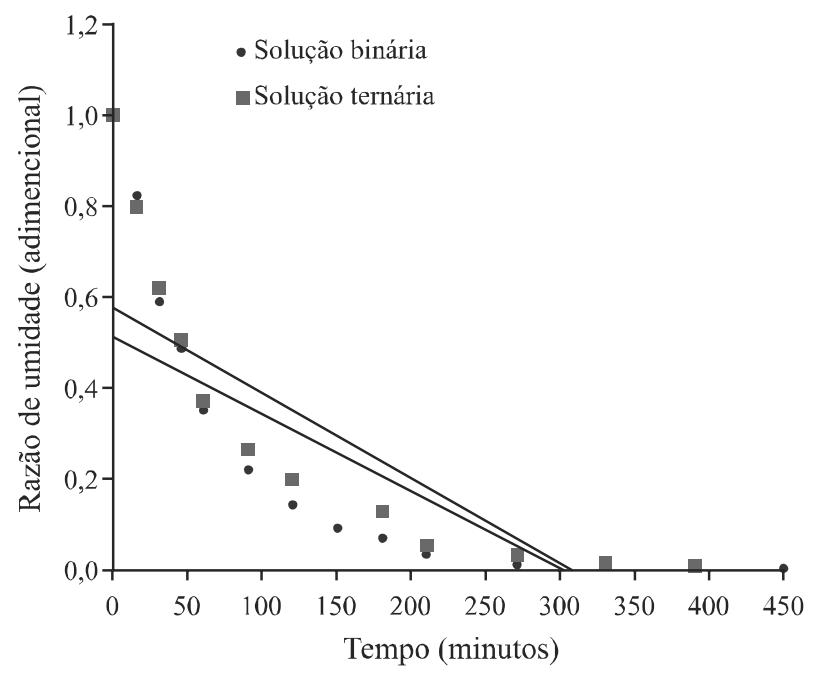

Analisando os parâmetros do modelo de Page, verifica-se que a constante $\mathrm{k}$ que representa a constante da taxa de secagem, foi maior nas amostras submetidas à desidratação em solução ternária e o contrário foi encontrado para a constante $n$, que reflete a resistência interna à secagem, em virtude da maneira a que essas condições externas foram impostas. $O$ fato desse modelo ser exponencial (natureza de todas as curvas de cinética de secagem) e ainda possuir dois parâmetros ajustáveis (k e n) favorece um melhor ajuste. Resultados semelhantes foram encontrados na secagem de mamão (EL-AOUAR; AZOUBEL; MURR, 2003), cenoura (DOYMAZ, 2004) e kiwi (SIMAL et al., 2005).

Tabela 1 - Parâmetros de ajuste dos modelos da cinética de secagem da polpa de cupuaçu em diferentes soluções, coeficientes de determinação $\left(\mathrm{R}^{2}\right)$ e erros percentuais médios $(\mathrm{P})$

\begin{tabular}{|c|c|c|c|c|c|c|c|}
\hline Modelo & Tratamento & ---------- & -------Parâr & ----------. & ------ & $\mathrm{R}^{2}(\%)$ & $\mathrm{P}(\%)$ \\
\hline \multirow{2}{*}{ Page } & Solução binária & \multicolumn{2}{|c|}{$\begin{array}{c}\mathrm{k} \\
0,015963 \\
\end{array}$} & \multicolumn{2}{|c|}{$\begin{array}{c}\mathrm{n} \\
1,004149\end{array}$} & 99,77 & 8,07 \\
\hline & Solução ternária & \multicolumn{2}{|c|}{$\begin{array}{c}\mathrm{k} \\
0,023316\end{array}$} & \multicolumn{2}{|c|}{$\begin{array}{c}\mathrm{n} \\
0,88670\end{array}$} & 99,64 & 7,32 \\
\hline \multirow{2}{*}{ Midilli et al } & Solução binária & $\begin{array}{c}\mathrm{k} \\
0,423670\end{array}$ & $\begin{array}{c}\mathrm{a} \\
0,786397\end{array}$ & $\begin{array}{c}b \\
-0,0017\end{array}$ & $\begin{array}{c}\mathrm{n} \\
0,000001\end{array}$ & 73,76 & 152,09 \\
\hline & Solução ternária & $\begin{array}{c}\mathrm{k} \\
0,168594\end{array}$ & $\begin{array}{c}a \\
0,687287\end{array}$ & $\begin{array}{c}b \\
-0,0019\end{array}$ & $\begin{array}{c}\mathrm{n} \\
0,000001\end{array}$ & 72,20 & 77,81 \\
\hline
\end{tabular}




\section{CONCLUSÕES}

Nas condições experimentais utilizadas neste estudo:

1. O menor tempo de secagem em estufa foi obtido para a polpa pré desidratada em solução binária, que reduziu os tempos do processo;

2. O modelo de Page apresenta o melhor ajuste aos dados experimentais, podendo ser utilizado para predição da cinética de secagem da polpa do cupuaçu.

\section{AGRADECIMENTOS}

Agradecimentos a CAPES e FAPEAM pelas bolsas de mestrado e doutorado, ao CNPq pela bolsa de produtividade e a FINEP pelos recursos financeiros.

\section{REFERÊNCIAS}

ANDRADE, S. A. et al. Desidratação osmótica do jenipapo (Genipa americana L.). Ciência e Tecnologia de Alimentos, v. 23, n. 02, p. 276-281, 2003.

ANDRADE, S. A. C. et al. Evaluation of water and sucrose diffusion coefficients during osmotic dehydration of jenipapo (Genipa americana L.). Journal of Food Engineering, v. 78, p. 551-555, 2007.

AZOUBEL, P. M.; MURR, F. E. X. Mass transfer kinetics of osmotic dehydration of cherry tomato. Journal of Food Engineering, v. 61, n. 03, p. 291-295, 2004.

BARONI, A. F. Propriedades mecânicas, termodinâmicas e de estado de tomate submetido à desidratação osmótica e secagem. 2004. 226 f. Tese (Doutorado em Engenharia de Alimentos) Universidade Estadual de Campinas, Campinas, 2004.

CORRÊA, J. L. G. et al. Desidratação osmótica de acerola (Malpighia emarginata D. C) - cinética de transferência de massa. Revista Ciência Agronômica, v. 39, n. 03, p. 403-409, 2008.

COSTA, M. C. et al. Conservação de polpa de cupuaçu [Theobroma grandiflorum (Willd. Ex Spreng.) Schum] por métodos combinados. Revista Brasileira de Fruticultura, v. 25, n. 02 , p. $213-215,2003$.

DIONELLO, R. G. et al. Secagem de fatias de abacaxi in natura e pré-desidratadas por imersão-impregnação: cinética e avaliação de modelos. Ciência e Tecnologia de Alimentos, v. 29, n. 01, p. 232-240, 2009.

DOYMAZ, I. Convective air drying characteristics of thin layer carrots. Journal of Food Engineering, v.61, n. 03, p. 359-364, 2004.

DOYMAZ, I. Drying kinetics of black grapes treated with different solutions. Journal of Food Engineering, v. 76, n. 02, p. 212-217, 2006.

EL-AOUAR, A. A.; AZOUBEL, P. M.; MURR, F. E. X. Drying kinetics of fresh and osmotically pretreated papaya (Carica papaya L.). Journal of Food Engineering, v. 59, p. 85-91, 2003.
FIOREZE, R. Princípios de secagem de produtos biológicos. João Pessoa: Editora UFPB, 2004. 229 p.

FITO, P. et al. Vacuum Impregnation and Osmotic Dehydration in Matrix Engineering. Application in Functional Fresh Food Development. Journal of Food Engineering, v. 49, n. 02/03, p. 175-183, 2001.

INSTITUTO ADOLFO LUTZ. Normas analíticas. In: Métodos químicos e físicos para análise de alimentos/ Coordenadores Odair Zenebon, Neus Sadocco Pascuet e Paulo Tiglea. São Paulo: Governo de São Paulo, 2008. 1020 p. Edição digital.

KARATHANOS, V. T.; BELESSIOTIS, V. G. Application of a thin-layer equation to drying data of fresh and semi-dried fruits. Journal of Agricultural Engineering Research, v. 74, n. 04, p. 355-361, 1999.

LENART, A. Osmo-convective drying of fruits and vegetables: Technology and application. Drying Technology, v. 14, n. 02, p. 391-413, 1996.

LIMA, E. E.; FIGUEIRÊDO, R. M. F.; QUEIROZ, A. J. M. Cinética de secagem de polpa de facheiro. Revista Brasileira de Produtos Agroindustriais, v. 9, n. 01, p. 17-28. 2007.

MASTRANTONIO, S. D. S.; PEREIRA, L. M.; HUBINGER, M. D. Mass transfer and difusion coeficient determination in osmotically dehydrated guavas. In: INTERNATIONAL DRYING SYMPOSIUM, 15., 2006, Budapest. Anais... Budapest: IDS, 2006. p. 860-870.

MIDILLI, A.; KUCUK, H.; YAPAR, Z. A new model for singlelayer drying. Drying Technology, v. 20, n. 07, p. 1503-1510, 2002.

PARK, K. J.; VOHNIKOZA, Z.; BROD, F. P. R. Evaluation of drying parameters and desorption isotherms of garden mint leaves (Mentha crispa L.). Journal of Food Engineering, v. 51, n. 03, p. 193-199, 2002.

QUEIROZ, A. J. M.; FIGUEIRÊDO, R. M. F.; CABRAL, M. F. P. Reologia de suco de cupuaçu. In: CONGRESSO BRASILEIRO DE ENGENHARIA AGRÍCOLA, 33., 2004, São Pedro. Anais... São Pedro: CONBEA, 2004. 1 CD-ROM.

ROMERO-PEÑA, L. M.; KIECKBUSCH, T. G. Influência de condições de secagem na qualidade de fatias de tomate. Brazilian Journal of Food Technology, v. 06, n. 01, p. 69-76, 2003.

SARAVACOS, G. D.; MAROULIS, Z. B. Transpor properties of food. New York: Marcel Dekker, 2001. 415p.

SERENO, A. M. et al. Prediction of Water Activity of Osmotic Solutions. Journal of Food Engineering, v. 49, n. 01, p. 103-114, 2001.

SIMAL, S. et al. Use of exponential, Page's and diffusional models to simulate the drying kinetics of kiwi fruit. Journal of Food Engineering, v. 66, N. 03, p. 323-328, 2005.

TONOM, R. V; BARONI, A. F.; HUBINGER, M. D. Estudo da desidratação osmótica de tomate em soluções ternárias pela metodologia de superfície de resposta. Ciência e Tecnologia de Alimentos, v. 26, n. 03, p. 715-723, 2006.

TSAMO, C. V. P. et al. Study of material transfer during osmotic dehydration of onion slices (Allium cepa) and tomato fruits (Lycopersicon esculentum). Lebensmittel-Wissenschaft und Technologie, v. 08, n. 05, p. 495-500, 2005. 